\title{
Análisis del título de anticuerpos en cerdas vacunadas contra peste porcina clásica a cuatro edades de gestación
}

\author{
Analysis of the antibody titre in sows vaccinated against classical swine fever at \\ four gestation ages
}

\author{
Jacqueline Alejandra Noboa Velástegui ${ }^{1,6}$, Alfredo Javier Acosta Batallas ${ }^{2}$, María \\ Augusta Chávez Larrea ${ }^{3}$, Rodolfo Fernández-Gómez ${ }^{3,5}$, Iván Patricio Yánez Ortiz ${ }^{4}$
}

\section{RESUMEN}

En el presente estudio se evaluó la respuesta inmune adquirida por vacunación contra la peste porcina clásica (PPC) en cerdas a cuatro edades distintas de gestación (70, 75, 80 y 90 días) empleando la vacuna Pest-Vac $\circledR$ (Pfizer). Se tomaron muestras de sangre 24 horas previas a la vacunación y 72 horas después del parto. Se determinó el nivel de anticuerpos mediante ELISA de bloqueo. No se encontró diferencia significativa entre los títulos de anticuerpos generados por la vacunación según la edad de gestación ni tampoco entre las muestras prevacunales y del posparto. Sin embargo, el efecto del número de parto, utilizado como covariable, presentó diferencia significativa $(\mathrm{p}<0.05)$ en el título de anticuerpos contra PPC.

Palabras clave: peste porcina clásica; virus de la peste porcina clásica; ELISA de bloqueo; Ecuador

\section{Abstract}

The immune response acquired by vaccination against classical swine fever (CSF) in sows at four gestational ages (70, 75, 80 and 90 days) was evaluated using the Pest-Vac $\mathbb{}$ vaccine (Pfizer). Blood samples were taken 24 hours before vaccination and 72 hours

\footnotetext{
${ }^{1}$ Universidad Regional Amazónica Ikiam, Tena, Ecuador

${ }^{2}$ Facultad de Medicina Veterinaria y Zootecnia, Universidad de São Paulo, São Paulo, Brasil

${ }^{3}$ Grupo de Investigación en Sanidad Animal y Humana (GISAH), Carrera Ingeniería en Biotecnología, Departamento de Ciencias de la Vida, Universidad de las Fuerzas Armadas ESPE, Sangolquí, Ecuador

${ }^{4}$ Subsecretaría de Producción Pecuaria, Ministerio de Agricultura y Ganadería, Quito, Ecuador

${ }^{5}$ Programa Prometeo, SENESCYT, Ecuador; Biophysic Solutions CA, Caracas, Venezuela

${ }^{6}$ E-mail: jacquenob@gmail.com
}

Recibido: 17 de septiembre de 2018

Aceptado para publicación: 7 de marzo de 2019 
after parturition. The level of antibodies was determined by blocking ELISA. No significant difference was found between the antibody titres generated by the vaccination according to gestational age nor between the pre-vaccination and post-partum samples. However, the effect of the number of parturitions, used as a covariate, presented a significant difference $(\mathrm{p}<0.05)$ in the antibody titres against PPC.

Key words: classical swine fever; classical swine fever virus; blocking ELISA; Ecuador

\section{INTRODUCCIÓN}

La peste porcina clásica (PPC), también conocida como cólera porcino o fiebre porcina clásica, es una epizootia de origen viral (VPPC). El virus pertenece a la familia Flaviviridae, género Pestivirus (Simmonds et al., 2017), los cuales infectan a cerdos y rumiantes, incluidos bovinos, ovinos, caprinos y rumiantes silvestres. Se transmiten a través del contacto con fluidos corporales y las infecciones pueden ser subclínicas o causar enfermedades entéricas, caracterizado por originar lesiones de carácter hemorrágico y de curso habitualmente fatal en las formas agudas, afectando a animales de todas las edades. La mortalidad y la morbilidad en la PPC suelen ser elevadas, siendo esta virosis considerada como una de las enfermedades porcinas de mayor relevancia a nivel mundial y de considerable impacto económico. La PPC es una enfermedad de declaración obligatoria ante la Organización Mundial de Sanidad Animal (OIE, 2009; Spickler, 2009; Blome et al., 2017).

Existen 16 marcadores únicos que corresponden a las once cepas del VPPC: Alfort187, Alfort Tubingen, Brescia, CAP, Glentorf, ALD, GPE, Chinese, C-strain, Riems, P97 (Zaberezhny et al., 1999). De estos, dos marcadores están en todas las cepas del VPPC, excepto en la cepa vacunal denominada CS, que es derivada de una cepa vacunal LK parental que ha sido atenuada por una serie de pasajes y clonación (Risatti et al., 2005). En el presente estudio se utilizó la vacuna Pest-Vac ${ }^{\circledR}$ (Pfizer), que corres- ponde a la cepa China, la cual se ha atenuado mediante pasajes sucesivos en células porcinas, a diferencia de otras vacunas, que son pasadas por células de otra especie.

En el caso del Ecuador, la PPC fue notificada por primera vez en la década de los 40 , y desde entonces ha ocasionado grandes pérdidas a la porcicultura nacional por su elevado índice de morbimortalidad, que va entre el 40 y $60 \%$, especialmente en las regiones de la sierra y la costa, y con menor proporción en la amazonia (AGROCALIDAD, 2012). A pesar de este problema, no se habían realizado estudios hasta el año 2013 para determinar la cepa circulante en el país, ni la respuesta inmune o la valoración de vacunas utilizadas para PPC. Actualmente, el Ecuador al igual que otros países, necesita apoyarse en la vacunación para erradicar la PPC, siguiendo las directrices para reconocimiento oficial emitidas en el Código Sanitario para los Animales Terrestres (OIE, 2013).

Con herramientas de biología molecular se ha podido detectar la diversidad de cepas del VPPC (Lu et al., 2017); sin embargo, el ELISA sería la prueba de oro (gold standard) para un proceso de diagnóstico y análisis de gran número de muestras. En el caso de la PPC, la OIE recomienda el ELISA como técnica de diagnóstico, no solo por su sensibilidad y especificidad, sino por ser de fácil adquisición, sencillez en su protocolo, facilidad de interpretación de resultados y por el gran número de muestras que se requiere analizar (OIE, 2009). El ELISA de bloqueo permite detectar anticuerpos contra el VPPC, pues los anticuerpos presentes en la muestra 
bloquean la unión de la peroxidasa que está conjugada a anticuerpos monoclonales específicos, provocando una disminución o aumento de coloración, dependiendo del título de anticuerpos contra el VPPC en animales infectados o no, respectivamente.

En tal sentido, el propósito de esta investigación fue generar información que sirva de base al Programa Nacional de Erradicación de Peste Porcina Clásica que lleva a cabo la Agencia de Regulación y Control Fito y Zoosanitario - AGROCALIDAD. El desarrollo y aplicación de protocolos y procedimientos estandarizados de vacunación y control, tanto a nivel regional como nacional, son procesos que mejoran la vigilancia sanitaria de la PPC.

\section{Materiales y Métodos}

El estudio fue realizado en la granja porcina «El Quinche», ubicada en la provincia de Pichincha, cantón Quito, parroquia El Quinche (Ecuador). Se utilizaron 36 cerdas Landrace $\mathrm{x}$ Yorkshire en gestación y clínicamente sanas. Las cerdas fueron distribuidas en forma aleatoria en 4 tratamientos de 9 repeticiones cada uno, de acuerdo con la época de vacunación contra PPC en el periodo de gestación: 70, 75, 80 y 90 días (Cuadro 1). Las cerdas en gestación no fueron homogenizadas por el número de parto, ya que se dio relevancia al número disponible de animales para cada tratamiento según el calendario de la granja, que vacunaban usualmente a los 70 o 75 días de gestación. La variable analizada fue el título de anticuerpos presente en las muestras de sangre a las 24 horas previas a la vacunación (prevacunal) y a las 72 horas del parto. Las muestras fueron tomadas de la vena yugular, en tubos con EDTA, trasladadas en cadena de frío hasta el laboratorio y almacenadas a una temperatura de $2-4{ }^{\circ} \mathrm{C}$. El análisis se realizó con suero sanguíneo una vez que las muestras fueron centrifugadas a $1500 \mathrm{rpm}$ por 5 minutos.
Cuadro 1. Distribución de los tratamientos $(n=9 /$ tratamiento) para analizar los títulos de anticuerpos vacunales medidos 24 horas previas a la vacunación y 72 horas después del parto

\begin{tabular}{cc}
\hline $\begin{array}{c}\text { Vacunación } \\
\text { (días de } \\
\text { gestación) }\end{array}$ & $\begin{array}{c}\text { Promedio de } \\
\text { partos/cerda }\end{array}$ \\
\hline 70 & 2.55 \\
75 & 3.44 \\
80 & 2.66 \\
90 & 3.77 \\
\hline
\end{tabular}

Los títulos de anticuerpos presentes en las muestras de suero sanguíneo se midieron con la prueba ELISA de bloqueo (CSFV Ab Test, IDEXX $\left.{ }^{\circledR}\right)$, que permite detectar la presencia de $\operatorname{IgG}$ contra el VPPC cepa China. El kit emplea anticuerpos monoclonales específicos contra el VPPC y el resultado se indica por el desarrollo de color. Se emplearon controles positivos y negativos por duplicado para validar el ensayo. La densidad óptica (DO) se midió a una longitud de onda de $450 \mathrm{~nm}$. Las muestras positivas dan un porcentaje de bloqueo $\geq 40 \%$ y las negativas $\leq 30 \%$; en tanto que se considera como sospechosa la muestra con valores intermedios. El porcentaje de bloqueo se calculó mediante la siguiente fórmula (IDEXX $\left.{ }^{\circledR}, 2013\right)$ : \% bloqueo $=\left(\left(\mu \mathrm{CN} \mathrm{A} \mathrm{A}_{450}\right.\right.$-Muestra $\left.\mathrm{A}_{450}\right) / \mu \mathrm{CN}$ $\left.\mathrm{A}_{450}\right) * 100$, donde $\mu \mathrm{CN} \mathrm{A}_{450}=$ promedio de la densidad óptica de los controles negativos leída a $450 \mathrm{~nm}$ de longitud de onda.

Los datos se analizaron mediante un análisis de covarianza, utilizando un diseño completamente al azar con medidas repetidas. La unidad experimental estuvo constituida por una cerda gestante, donde los factores en estudio fueron la época de vacunación durante la gestación y el periodo de toma de muestra, utilizando como covariable el número de parto de las cerdas. El modelo estadístico fue $\mathrm{Y}_{\mathrm{ijk}}=\mathrm{m}+\mathrm{T}_{\mathrm{i}}+\mathrm{M}_{\mathrm{ij}}+\mathrm{P}_{\mathrm{k}}+$ 
Cuadro 2. Título de anticuerpos vacunales frente al VPPC generados por la vacuna PestVac ${ }^{\circledR}$ aplicada en cuatro edades de gestación y medidos 24 horas previas a la vacunación y 72 horas después del parto

\begin{tabular}{|c|c|c|c|c|c|}
\hline \multirow{2}{*}{ Tratamiento $^{1}$} & \multicolumn{4}{|c|}{$\%$ Bloqueo } & \multirow{2}{*}{$\begin{array}{c}\text { Contraste (pre- } \\
\text { vacunal vs. } \\
\text { posparto) }\end{array}$} \\
\hline & Prevacunal & $\mathrm{DE}^{2}$ & Posparto & $\mathrm{DE}$ & \\
\hline V70 & $69.32^{\mathrm{a}}$ & 23.32 & $73.39^{\mathrm{a}}$ & 18.01 & $\mathrm{NS}^{4}$ \\
\hline V75 & $70.60^{\mathrm{a}}$ & 32.80 & $77.76^{\mathrm{a}}$ & 21.41 & NS \\
\hline V80 & $59.26^{\mathrm{a}}$ & 20.99 & $52.97^{\mathrm{a}}$ & 21.05 & NS \\
\hline V90 & $75.66^{\mathrm{a}}$ & 13.92 & $63.93^{\mathrm{a}}$ & 18.08 & NS \\
\hline SEM $^{3}$ & \multicolumn{2}{|l|}{7.73} & \multicolumn{2}{|c|}{7.02} & \\
\hline
\end{tabular}

Letras distintas indican diferencia significativa $(p<0.05)$

${ }^{1}$ V70; V75, V80 y V90: Vacunación a los 70, 75, 80 y 90 días de gestación; V75 = testigo

${ }^{2} \mathrm{DE}=$ Desviación estándar

${ }^{3} \mathrm{SEM}=$ Error estándar de la media

${ }^{4}$ NS: No significativo

$(\mathrm{TP})_{\mathrm{ik}}+\gamma \mathrm{X}_{\mathrm{ij}}+\mathrm{e}_{\mathrm{ijk}}$, donde $\mathrm{Y}_{\mathrm{ijk}}=$ título de anticuerpos, $\mathrm{m}=$ media general, $\mathrm{T}=$ tratamientos, $\mathrm{M}_{\mathrm{ij}}=$ efecto aleatorio de la madre, $\mathrm{P}_{\mathrm{k}}=$ periodo de toma de muestra, $(\mathrm{TP})_{\mathrm{ik}}=$ interacción del tratamiento con el periodo de toma de muestra, $\gamma \mathrm{X}_{\mathrm{ij}}=$ coeficiente de covarianza, $\mathrm{ye}_{\mathrm{ijk}}=$ error aleatorio.

Los datos obtenidos durante el proceso experimental fueron analizados utilizando el procedimiento de Modelos Lineales Mixtos (MIXED) del paquete estadístico SAS (SAS, 2015). Los rangos de significación fueron establecidos mediante la prueba de Tukey al $5 \%$ para determinar las diferencias estadísticas en las diferentes épocas de vacunación durante la gestación y en los periodos de toma de muestras, así como en su interacción. Las medias se ajustaron por mínimos cuadrados.

\section{Resultados y Discusión}

No se encontró diferencias significativas ( $p>0.05$ ) entre los porcentajes de bloqueo de los anticuerpos contra PPC en cerdas vacunadas en cuatro edades de gestación, tanto en el muestreo prevacunal como en el muestreo a las 72 horas posparto (Cuadro 2). Este resultado da a entender que una vez que las cerdas han sido vacunadas por reiteradas ocasiones o dentro de estos días de gestación (70 a 90 días) no presentan respuestas inmunológicas diferentes frente a la vacuna. Qiu et al. (2006) mencionan que una sola aplicación de la vacuna en algunos animales es suficiente para dar inmunidad de por vida; por lo tanto, si las cerdas de este estudio ya han sido previamente inmunizadas y revacunadas en cada gestación, no se garantiza que haya un incremento de anticuerpos en cada nueva aplicación de la vacuna.

Es posible que esta respuesta de por vida en una sola aplicación se deba al tipo de vacuna que se está empleando. Suradhat et al. (2007) indican que la vacuna con virus vivo atenuado de cepa China es una de las vacunas que mayor protección clínica y vírica proporciona. De igual forma, Muñoz-González et al. (2015) indican que la vacuna persiste y se replica en las tonsilas de los animales por 30 días. 
En una investigación realizada por Báez et al. (1995), cerdas sin historia previa de vacunación contra el VPCC, fueron vacunadas a los 30, 60 y 90 días de gestación, sin que se encontrase diferencia estadística en los títulos de anticuerpos vacunales entre estos grupos.

No se conoce el número de revacunaciones necesarias o el límite para crear una inmunidad permanente. Iuminova et al. (2002), evaluando la vacunación contra el virus de la parotiditis (paperas), encontraron que el nivel de anticuerpos luego de la revacunación aumenta cuando los anticuerpos iniciales o previo a una segunda dosis estuvieron bajos. Así mismo, Ming et al. (2011) evaluaron los títulos de anticuerpos utilizando la vacuna Pest-Vac $\AA$, encontrando un buen título de anticuerpos vacunales en plasma como en calostro a las 72 horas posparto. Estas investigaciones pueden explicar la razón de no haber encontrado diferencias significativas en este estudio, ya que en aquellos estudios los animales han partido de una concentración negativa de anticuerpos.

Es importante considerar el tiempo de gestación al establecer un protocolo de inmunización, porque este puede generar estrés en las cerdas, más aún en el último tercio (Step et al., 2009). En el presente estudio no se analizaron los parámetros reproductivos, pero se puede indicar que no hubo abortos, lechones nacidos muertos, ni fetos momificados. En este sentido, Fonseca et al. (2011) recomiendan vacunar a las cerdas hasta el segundo tercio de gestación para que no haya problemas en la gestación a término.

El objetivo del presente estudio se enfocó en evaluar cómo varía la respuesta inmune a la vacuna cuando es aplicada en diferentes edades de gestación. Por otra parte, el efecto del número de parto de las cerdas utilizado como covariable presentó diferencia significativa $(p<0.05)$ en el título de anticuerpos contra PPC. Como en el estado de gestación debe existir «tolerancia» al feto, se necesita un cierto grado de inmunosupresión y al mismo tiempo el organismo de la cerda debe ser capaz de combatir posibles infecciones. Para mantener con éxito la gestación debe haber un cambio de perfil de citocinas de Th1 a Th2; es decir, una respuesta que sea de tipo celular o de tipo humoral mediante la generación de anticuerpos, respectivamente. Por lo tanto, de acuerdo con el tipo de vacuna que se emplee, generará una respuesta inmune completa (celular y humoral) (Yan, 2012). Como los linfocitos Th2 son necesarios para una respuesta con linfocitos $\mathrm{B}$, dependientes de los linfocitos $\mathrm{T}$, podría dar a entender el por qué no ha habido una diferencia significativa en cuanto a los tratamientos por el nivel de inmunosupresión en estado de gestación, pero sí en el número de parto, aunque sin determinar en cuál de los tratamientos. Se podría decir que una cerda con varios partos está más familiarizada con el estrés de la gestación a diferencia de una cerda primeriza o de pocos partos.

Por otro lado, el Proyecto de Control y Erradicación de Peste Porcina Clásica ejecutado por la Agencia de Regulación y Control Fito y Zoosanitario - AGROCALIDAD, en su apartado «Guía Técnica del Vacunador de Peste Porcina Clásica», recomienda la aplicación de la vacuna a partir de los 90 días de gestación o en la primera semana de lactancia (AGROCALIDAD, 2014). Sin embargo, no se detalla el tipo de vacuna, factor a tomar en cuenta por el tipo de células en las que se hace el pasaje de los virus vivos para su atenuación. Así mismo, la vía de administración de la vacuna es un factor crítico para generar una buena respuesta inmunológica (Matías et al., 2017).

Es importante señalar que, mediante Resolución N ${ }^{\circ} 1950$ de la Secretaría General de la Comunidad Andina, se aprobó el Plan Operativo del Programa Subregional para la Prevención, Control y Erradicación de la Peste Porcina Clásica (PPC), periodo 2017-2018 (Resolución $\mathrm{N}^{\circ} 1950,2017$ ), apoyado por la FAO y la OIE. En este sentido, Colombia y 
Perú están teniendo excelentes resultados; de hecho, en 2017 la OIE entregó a Colombia el certificado que acredita a la principal zona productora de cerdos, que incluyen los departamentos de Chocó, Caldas, Quindío, Risaralda, Valle del Cauca y algunos municipios de Antioquia y Cauca, como libre de PPC. Por su parte, Perú tiene en práctica un plan masivo de vacunación, información y educación sanitaria sobre todo lo que atañe a la PPC.

\section{Conclusiones}

- La vacunación a cerdas entre los $70 \mathrm{y}$ 90 días de gestación y positivas contra anticuerpos PPC de vacunaciones previas, no genera títulos de anticuerpos vacunales significativamente diferentes.

- La revacunación en cada gestación no garantiza una mejor inmunización.

- El número de partos por cerda influye sobre el título de anticuerpos, posiblemente debido a un proceso de adaptación del sistema inmune de la cerda al estado de gestación.

\section{Agradecimientos}

Se agradece la asesoría científica de la Universidad de las Fuerzas Armadas, la capacitación técnica de la Agencia de Regulación y Control Fito y Zoosanitario AGROCALIDAD, y la ayuda y financiamiento de la granja porcina «El Quinche».

\section{Literatura Citada}

1. AGROCALIDAD. 2012. Proyecto de control y erradicación de peste porcina clásica por zonificación en el Ecuador. [Internet]. Disponible en: http:// www.agrocalidad.gob.ec/documentos/ ppc/d1.pdf
2. AGROCALIDAD. 2014. Guía técnica del vacunador de peste porcina clásica. [Internet]. Disponible en: http:// www.agrocalidad.gob.ec/documentos/ ppc/6.pdf

3. Báez U, Coba M, Anaya A, Correa P, Rosales C. 1995. Inocuidad del virus vacunal PAV-250 contra la fiebre porcina clásica (FPC) en cerdas en celo y gestantes, sin antecedentes de vacunación. Tec Pecu Méx 33: 135-147.

4. Blome S, Staubach C, Henke J, Carlson J, Beer M. 2017. Classical swine fever - an updated review. Viruses 9: E86. doi: 10.3390/v9040086

5. Fonseca O, Domínguez, P, Rodríguez MP, González R, Reyes M, Abeledo MA, Suárez M, et al. 2011. Comportamiento reproductivo de cerdas gestantes inmunizadas con un candidato vacunal de subunidad E2 contra el Virus de la peste porcina clásica. Rev Salud Anim 33: 203-205.

6. IDEXX®. 2013. Classical swine fever virus (CSFV) antibody test kit. [Internet]. Disponible en: http://www.dimune.com/ assets/06-43260-06-insert-csfv-ab.pdf

7. Iuminova NV, Aleksander SK, Liashchenko VA, Sidorenko ES. 2002. Efficiency of revaccination against epidemic parotitis and immunological safety. Vopr Viruso 47: 44-45 [En ruso].

8. LuX, Shi X, Wu G, Wu T, Qin R, Wang Y. 2017. Visual detection and differentiation of classic swine fever virus strains using nucleic acid sequencebased amplification (NASBA) and Gquadruplex DNAzyme assay. Sci Rep 7: 44211. doi: 10.1038/srep44211

9. Matías J, Berzosa M, Pastor Y, Irache JM, Gamazo C. 2017. Maternal vaccination. Immunization of sows during pregnancy against ETEC infections. Vaccines 5: E48. doi: 10.3390/vaccines5040048

10. Ming $Y$, Allison J, Lehrbach $P$, Navasakuljinda W, Poolperm P. 2011. Efficacy of a commercial swine fever vaccine against a virulent Thailand 
isolate of classical swine fever virus. In: $5^{\text {th }}$ Asian Pig Veterinary Society Congress. Thailand.

11. Muñoz-González S, Perez-Simó M, Muñoz M, Bohorquez JA, Rosell R, Summerfield A, Domingo M. 2015. Efficacy of a live attenuated vaccine in Classical Swine Fever Virus postnatally persistently infected pigs. Vet Res 46: 78. doi: 10.1186/s13567-015-0209-9

12. [OIE] World Organisation for Animal Health. 2009. Classical Swine Fever (hog cholera). [Internet]. Disponible en: http://www.oie.int/fileadmin/Home/eng/ Animal_Health_in_the_World/docs/pdf/ Dis e a s e c a rd s / C L A S S I CAL_SWINE_FEVER.pdf

13. [OIE] Organización Mundial de Sanidad Animal. 2013. Código sanitario para los animales terrestres. [Internet]. Disponible en: https://www.oie.int/doc/ ged/D12823.pdf

14. Qiu H, Shen R, Tong G. 2006. The lapinized Chinese strain vaccine against classical swine fever virus: a retrospective review spanning half a century. Agric Sci China 5: 1-14. doi: 10.1016/S1671-2927(06)60013-8

15. Resolución $N^{o} 1950$. Adopcioìn del Plan Operativo y Cronograma de Ejecucioìn del Programa Subregional Andino de Prevencioìn, Control y Erradicacioìn de la Peste Porcina Claìsica, 2017 - 2018, Lima, Perú, 28 de septiembre de 2017.

16. Risatti GR, Borca MV, Kutish GF, Lu Z, Holinka LG, French RA, Tulman ER, et al. 2005. The E2 glycoprotein of classical swine fever virus is a virulence determinant in swine. J Virol 79: 37873796. doi: 10.1128/JVI.79.6.37873796.2005
17. SAS. Statystical Analysis System. 2015. SAS/STAT ${ }^{\circledR}$ user's guide v. 9.4. Cary, NC: SAS Institute Inc.

18. Simmonds P, Becher P, Bukh J, Gould EA, Meyers G, Monath T, Muerhoff $S$, et al. 2017. ICTV virus taxonomy profile: Flaviviridae. J Gen Virol 98: 2-3. doi: 10.1099/jgv.0.000672

19. Spickler AR. 2009. Peste porcina clásica. [Internet]. Disponible en: http:// www.cfsph.iastate.edu/Factsheets/es/ classical_swine_fever-es.pdf

20. Step DL, Krehbiel CR, Burciaga-Robles LO, Holland BP, Fulton $R W$, Confer AW, Bechtol DT, et al. 2009. Comparison of single vaccination versus revaccination with a modified-live virus vaccine containing bovine Herpesvirus1, Bovine Viral Diarrhea Virus (types 1a and 2a), Parainfluenza type 3 virus, and Bovine Respiratory Syncytial Virus in the prevention of bovine respiratory disease in cattle. J Am Vet Med Assoc 235: 580 587. doi: 10.2460/javma.235.5.580

21. Suradhat S, Damrongwatanapokin S, Thanawongnuwech $R$. 2007. Factors critical for successful vaccination against classical swine fever in endemic areas. Vet Microbiol 119: 1-9. doi: 10.1016/ j.vetmic.2006.10.003

22. Yan W. 2012. Impact of prenatal stress and adulthood stress on immune system: a review. Biomed Res-Tokyo 23: 315-320.

23. Zaberezhny AD, Grebennikova TV, Kurinnov VV, Tsybanov SG, Vishnyakov IF, Biketov SF, Aliper TI, et al. 1999. Differentiation between vaccine strain and field isolates of classical swine fever virus using polymerase chain reaction and restriction test. Deut Tierarztl Woch 106: 394-397. 